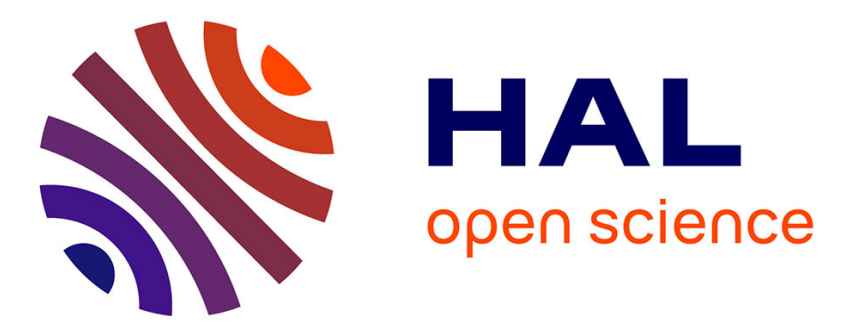

\title{
Electrostatic Steepening of Whistler Waves
}

Ivan Y. Vasko, Oleksiy V. Agapitov, Forrest S. Mozer, John W. Bonnell, Anto V. Artemyev, Vladimir V. Krasnoselskikh, Yuguang Tong

\section{To cite this version:}

Ivan Y. Vasko, Oleksiy V. Agapitov, Forrest S. Mozer, John W. Bonnell, Anto V. Artemyev, et al.. Electrostatic Steepening of Whistler Waves. Physical Review Letters, 2018, 120 (195101), 10.1103/PhysRevLett.120.195101 . insu-01897794

\section{HAL Id: insu-01897794 https://hal-insu.archives-ouvertes.fr/insu-01897794}

Submitted on 10 Dec 2019

HAL is a multi-disciplinary open access archive for the deposit and dissemination of scientific research documents, whether they are published or not. The documents may come from teaching and research institutions in France or abroad, or from public or private research centers.
L'archive ouverte pluridisciplinaire HAL, est destinée au dépôt et à la diffusion de documents scientifiques de niveau recherche, publiés ou non, émanant des établissements d'enseignement et de recherche français ou étrangers, des laboratoires publics ou privés. 


\title{
Electrostatic Steepening of Whistler Waves
}

\author{
I. Y. Vasko, ${ }^{1,}{ }^{*}$ O. V. Agapitov, ${ }^{1,3}$ F. S. Mozer, ${ }^{1}$ J. W. Bonnell, ${ }^{1}$ A. V. Artemyev, ${ }^{4,2}$ V. V. Krasnoselskikh, ${ }^{5}$ and Y. Tong ${ }^{1}$ \\ ${ }^{1}$ Space Sciences Laboratory, University of California, Berkeley, California 94720, USA \\ ${ }^{2}$ Space Research Institute RAS, Moscow 117997, Russia \\ ${ }^{3}$ National Taras Shevchenko University of Kyiv, Kyiv 01033, Ukraine \\ ${ }^{4}$ University of California in Los Angeles, Los Angeles, California 90095, USA \\ ${ }^{5}$ LPC2E, University of Orleans, Orléans 45100, France
}

(Received 30 January 2018; revised manuscript received 26 March 2018; published 9 May 2018)

\begin{abstract}
We present surprising observations by the NASA Van Allen Probes spacecraft of whistler waves with substantial electric field power at harmonics of the whistler wave fundamental frequency. The wave power at harmonics is due to a nonlinearly steepened whistler electrostatic field that becomes possible in the two-temperature electron plasma due to the whistler wave coupling to the electron-acoustic mode. The simulation and analytical estimates show that the steepening takes a few tens of milliseconds. The hydrodynamic energy cascade to higher frequencies facilitates efficient energy transfer from cyclotron resonant electrons, driving the whistler waves, to lower energy electrons.
\end{abstract}

DOI: 10.1103/PhysRevLett.120.195101

Whistler waves play a fundamental role in electron acceleration in space [1,2], solar wind [3], and astrophysical [4] plasmas, and they continuously stimulate laboratory plasma experiments $[5,6]$. In particular, whistler waves are one of the factors controlling the dynamics of the Van Allen radiation belts [7], where they are generated via the cyclotron resonant instability with regularly injected $\sim 10 \mathrm{keV}$ anisotropic electrons [8]. Whistler waves mediate the energy of the injected electrons to higher and lower energy electrons via the resonant interaction, resulting in electron acceleration up to relativistic energies $[1,2]$ and electron losses to the atmosphere [9].

Whistler waves are typically observed in the form of quasimonochromatic wave packets propagating quasiparallel to the background magnetic field [10]. The whistler wave field is decomposed into the electrostatic field along the wave vector $\mathbf{k}$ and elliptically polarized electromagnetic field perpendicular to $\mathbf{k}$ [11]. Whistler waves are fundamentally different from compressible sound waves in fluids and plasmas $[12,13]$ in that the whistler electrostatic field has been argued not to steepen [14]. In accordance, the reported whistler waves in the Van Allen radiation belts typically have quasisinusoidal waveforms even at the highest observed amplitudes [15]. Slightly nonsinusoidal electric field waveforms have been attributed to electrons trapped within the whistler electrostatic field [16].

In this Letter, we present whistler waves with a surprisingly significant electric field power at harmonics of the fundamental frequency that are due to the highly nonsinusoidal waveform of the whistler electrostatic field (also recently reported in Ref. [17]). The wave energy cascade to higher frequencies is due to the classical hydrodynamic steepening that becomes possible in the two-temperature electron plasma.

The twin NASA Van Allen Probes spacecrafts, launched on August 30, 2012 into the Van Allen radiation belts, provide wave and particle measurements with unprecedent time resolution. We present Van Allen Probe A measurements on May 1, 2013 near Earth's magnetic dipole equator (at magnetic latitude of about $1^{\circ}$ ) at the geocentric radial distance of 5.5 Earth radii. During the considered time interval, the background magnetic field is about $87 \mathrm{nT}$, while the electron density inferred from estimate of the upper hybrid frequency is about $3.2 \mathrm{~cm}^{-3}$. The electron cyclotron and plasma frequencies are $f_{c} \sim 2.5 \mathrm{kHz}$ and $f_{p} \sim 16 \mathrm{kHz}$.

Figure 1 shows one second of the waveform of whistler waves continuously present for more than ten seconds around 11:27:25 UT. The left panels show the waveform in the coordinate system related to the background magnetic field. Surprisingly, the waveform of the parallel electric field is highly nonsinusoidal, in contrast to the other electric and magnetic field components. The middle panels present $30 \mathrm{~ms}$ of the waveform in the coordinate system with the $Z$ axis along the whistler wave vector that is the direction delivering minimum to the root mean square of div $\mathbf{B}$ [18]. In this coordinate system, the whistler wave field is decomposed into the electrostatic field $E_{z}$ and the electromagnetic field in the $X Y$ plane (Fig. 2).

The selected $30 \mathrm{~ms}$ whistler wave packet propagates at the wave normal angle $\theta \sim 15^{\circ}$. The electromagnetic fields have quasisinusoidal waveforms and their spectra are peaked at $f \sim 450 \mathrm{~Hz}$ that is about $0.2 f_{c}$. There is a good correlation between $E_{y}$ and $B_{x}$, in accordance with Faraday's law, and $E_{y} / B_{x}$ provides the phase velocity 

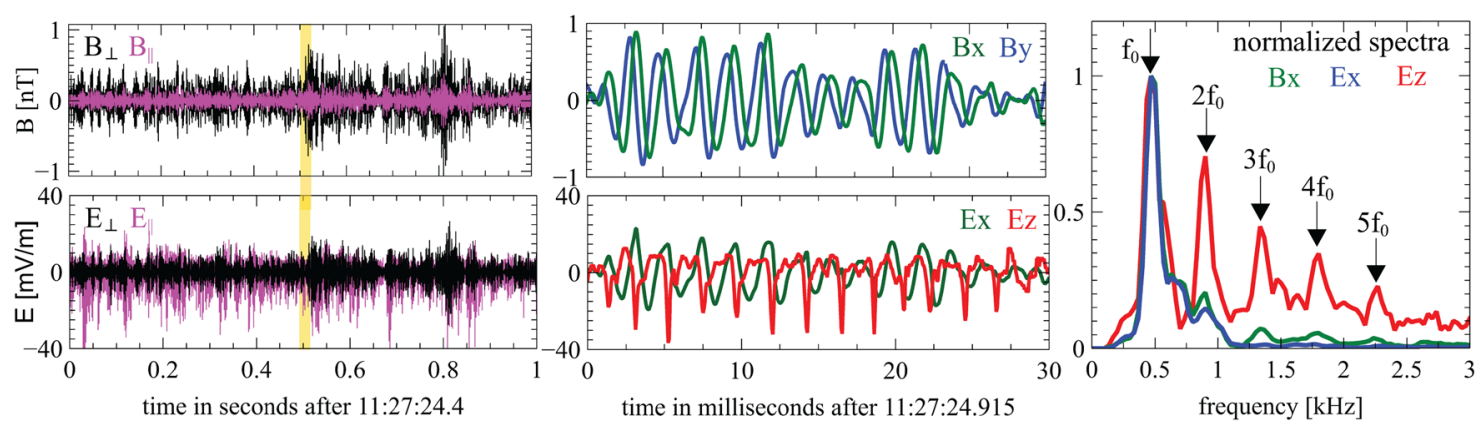

FIG. 1. The left panels present electric and magnetic field waveforms measured with cadence 16384 samples/s by the Electric Field Instrument [19] and Electric and Magnetic Field Instrument Suite and Integrated Science [20] aboard the Van Allen Probe A on May 1, 2013. The waveforms are in the coordinate system related to the background (DC) magnetic field: $E_{\perp}, B_{\perp}$ are one of the electric and magnetic field components perpendicular to the background magnetic field, while $E_{\|}, B_{\|}$are parallel to it. The middle panels present the waveform over $30 \mathrm{~ms}$ in the natural coordinate system shown in Fig. 2. The right panel presents the spectrum of the electrostatic $E_{z}$ and electromagnetic $E_{x}, B_{x}$ fields for the selected $30 \mathrm{~ms}$.

estimate of about $15000 \mathrm{~km} / \mathrm{s}$ that is in reasonable agreement with $c\left[f\left(f_{c} \cos \theta-f\right) / f_{p}^{2}\right]^{1 / 2} \sim 17500 \mathrm{~km} / \mathrm{s}$ from the dispersion relation in a cold plasma [11]. The electrostatic field $E_{z}$ has a highly nonsinusoidal waveform with pronounced negative electric field spikes showing up in the wave spectrum as electric field power at harmonics of $\sim 450 \mathrm{~Hz}$. Van Allen Probe measurements show that the whistler waves are associated with the presence of the twotemperature electron population.

Figure 3 presents cuts of the electron spectrum corresponding to fluxes of electrons with pitch angles around $0^{\circ}$ and $90^{\circ}$. The energy spectrum is anisotropic above a few $\mathrm{keV}$, as required to excite whistler waves via the cyclotron resonant instability [8]. The high-energy part of the electron spectrum is fitted to the $\kappa$ - distribution, resulting in a

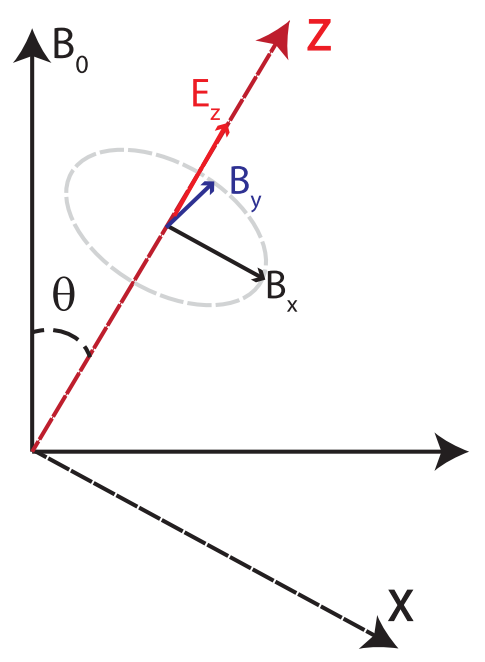

FIG. 2. The coordinate system with the $Z$ axis along the wave vector directed at a wave normal angle $\theta$, with respect to the background magnetic field $\mathbf{B}_{0}$. The whistler wave field is decomposed into the electrostatic field $E_{z}$ and the electromagnetic field in the $X Y$ plane. density $\sim 2 \mathrm{~cm}^{-3}$ and a temperature $\sim 4.3 \mathrm{keV}$. The spectrum is not resolved below $100 \mathrm{eV}$ because of contamination by photoelectrons. The total electron density of about $3.2 \mathrm{~cm}^{-3}$ indicates the density of the low-energy electron population (below $\sim 1 \mathrm{keV}$ ) of about $1.2 \mathrm{~cm}^{-3}$. The temperature of the low-energy population is below a few hundred $\mathrm{eV}$.

We address the whistler wave dynamics in the twotemperature electron plasma using the hydrodynamic and Maxwell equations. Because of the high whistler wave frequency, ions can be considered as immobile charge neutralizing background [11]. In the coordinate system

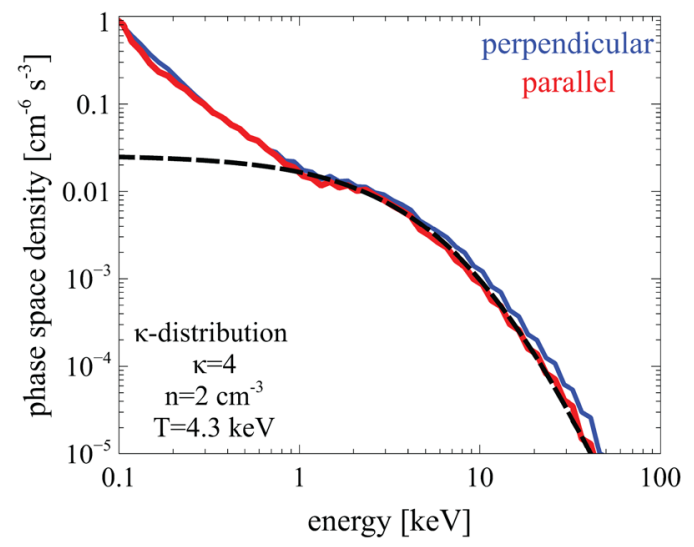

FIG. 3. The spectrum (phase space density) of electrons with pitch angles around $0^{\circ}$ (streaming parallel to the background magnetic field) and around $90^{\circ}$ (streaming perpendicular to it) computed by converting the fluxes measured by the Helium Oxygen proton electron (HOPE) detector [21] over 10 seconds, around 11:27:25 UT. The high-energy part of the spectrum is fitted to $\mathcal{F}(\mathcal{E})=n \mathcal{C}_{\kappa}\left(m / 2 \pi \kappa \mathcal{E}_{0}\right)^{3 / 2}\left[1+\mathcal{E} / \kappa \mathcal{E}_{0}\right]^{-(\kappa+1)}$, where $\mathcal{C}_{\kappa}=$ $\Gamma(\kappa+1) / \Gamma(\kappa-1 / 2), n$ is the density, and $T=2 \kappa \mathcal{E}_{0} /(2 \kappa-3)$ is the temperature. The fitting parameters $\kappa, n$, and $T$ are presented in the panel. 
shown in Fig. 2, the hydrodynamic equations for the two electron populations can be written as

$$
\begin{aligned}
\frac{d}{d t}\left(u_{j}-\frac{e A_{x}}{m c}\right)= & -2 \pi f_{c} v_{j} \cos \theta, \\
\frac{d}{d t}\left(v_{j}-\frac{e A_{y}}{m c}\right)= & 2 \pi f_{c}\left(u_{j} \cos \theta+w_{j} \sin \theta\right), \\
\frac{d w_{j}}{d t}= & \frac{e}{m} \frac{\partial \Phi}{\partial z}-\frac{1}{m n_{j}} \frac{\partial\left(T_{j} n_{j}\right)}{\partial z}-2 \pi f_{c} v_{j} \sin \theta \\
& -\frac{e}{m c}\left(u_{j} \frac{\partial A_{x}}{\partial z}+v_{j} \frac{\partial A_{y}}{\partial z}\right), \\
\frac{d n_{j}}{d t}= & -n_{j} \frac{\partial w_{j}}{\partial z}, \quad \frac{d}{d t} \equiv \frac{\partial}{\partial t}+w_{j} \frac{\partial}{\partial z},
\end{aligned}
$$

where $j=l, h$ corresponds to the low- and highenergy populations, $n_{j},\left(u_{j}, v_{j}, w_{j}\right)$ and $T_{j}$ are electron densities, bulk velocities and temperatures, and $-e$ and $m$ are the electron charge and mass. The Maxwell equations for the electrostatic and vector potentials are $\partial^{2} \Phi / \partial z^{2}=4 \pi e\left(\sum_{j} n_{j}-n_{0}\right) \quad$ and $\quad \partial^{2} \mathbf{A} / \partial z^{2}=$ $(4 \pi e / c) \sum_{j}\left(n_{j} u_{j} \hat{x}+n_{j} v_{j} \hat{y}\right)$, where $n_{0}$ is the unperturbed electron density and the displacement current is neglected because $\partial^{2} / \partial t^{2} \ll c^{2} \partial^{2} / \partial z^{2}$. We neglect the thermal spread of the low-energy population as not qualitatively affecting the whistler wave dynamics. The high-energy population is assumed to be isothermal $T_{h}=$ const as in the theory of ion-acoustic waves [13], because its thermal velocity is higher than the whistler wave phase velocity. By linearizing the equations we obtain the dispersion relations of linear waves propagating in the observed plasma.

Figure 4 shows that the whistler wave dispersion relation is not affected by the thermal spread, and it coincides with the cold dispersion relation, $f \approx f_{c} \cos \theta k^{2} c^{2} /$ $\left(k^{2} c^{2}+4 \pi^{2} f_{p}^{2}\right)$ [11]. The two-temperature electron plasma supports electrostatic electron-acoustic waves, the dispersion relation of electron-acoustic waves at long wavelengths is $2 \pi f \approx k v_{E A} \cos \theta$, where $v_{E A}=$ $\left(T_{h} / m\right)^{1 / 2}\left(n_{l} / n_{0}\right)^{1 / 2}$ is the electron-acoustic velocity $[22,23]$. Whistler and electron-acoustic waves turn out to be coupled in the crossover points at $f \sim 0.2 f_{c}$ and $\sim 0.8 f_{c}$. The most pronounced effect around the crossover frequencies is seen in the electron compressibility that is the ratio of amplitudes of compressional $w_{j}$ and noncompressional bulk velocities. The high- and low-energy electron populations become highly compressible around the crossover frequencies, although the full electron compressibility remains negligible because compressional oscillations of the high- and low-energy electron populations are in antiphase.

We address the nonlinear evolution of whistler waves by solving the hydrodynamic and Maxwell equations using the energy conserving numerical scheme based on the fast

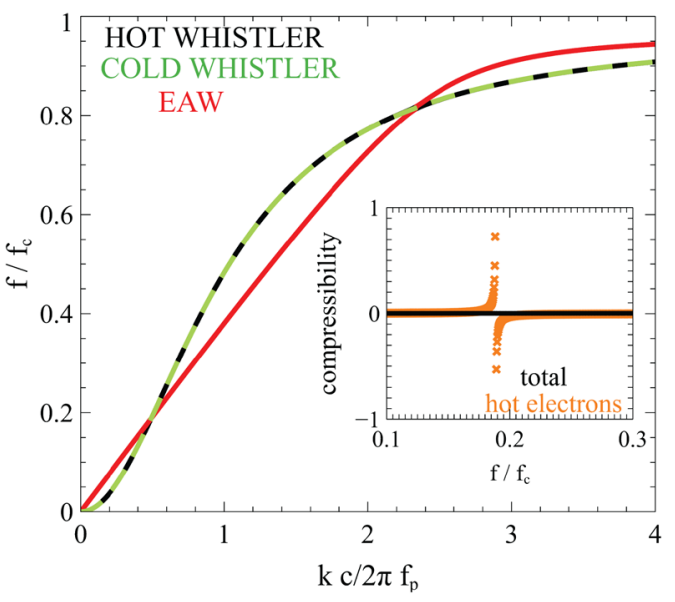

FIG. 4. The dispersion relation of linear waves propagating in the observed two-temperature electron plasma below the electron cyclotron frequency and above the low-hybrid frequency (ions are considered as immobile charge neutralizing background). The dispersion relations are computed for the wave normal angle $\theta=15^{\circ}$. The dashed green curve shows the dispersion relation of whistler waves in a cold plasma computed by setting zero thermal spread of the high-energy population. The small panel presents the compressibility of the high-energy population and the full electron compressibility defined as the ratio of amplitudes of the compressional velocities $w_{h}$ and $w_{0}$ and noncompressional bulk velocity $\left(u_{0}^{2}+v_{0}^{2}\right)^{1 / 2}$, where $\left(u_{0}, v_{0}, w_{0}\right)=\sum_{j} n_{j}\left(u_{j}, v_{j}, w_{j}\right) / n_{0}$ is the full electron bulk velocity.

Fourier transform previously used for the analysis of the steepening of electron-acoustic waves [24]. The initial condition is a monochromatic whistler wave of a realistic amplitude. We have found that whistler waves with the frequency far from the crossover frequencies remain undisturbed in accordance with the previous simulations [14]. On the contrary, whistler waves with the frequency around the crossover frequencies exhibit a fundamentally different behavior.

Figure 5 presents the evolution of a whistler wave with the frequency $f_{0}$ around the first crossover frequency corresponding to the wave number $k_{0} \sim \pi f_{p} / c$. The evolution of the electrostatic potential exhibits signatures of the classical hydrodynamic steepening (overtaking) inherent to sound waves in fluids and plasmas $[12,13]$. The steepening produces the negative electric field spikes in the electrostatic component. The spikes become quite pronounced after about $40 \mathrm{~ms}$ and resemble those in observations. The magnetic field $B_{x}$ and the other electromagnetic components are slightly distorted in accordance with the presence of some wave power at the harmonics in the observed spectra (Fig. 1).

The steepening of the whistler electrostatic field is identical to the steepening of electron-acoustic waves [24]. In fact, the physics of the effect are equivalent. An initially monochromatic sufficiently long wavelength electronacoustic wave $(f, k)$ produces harmonics $(\ell f, \ell k)$ that are 


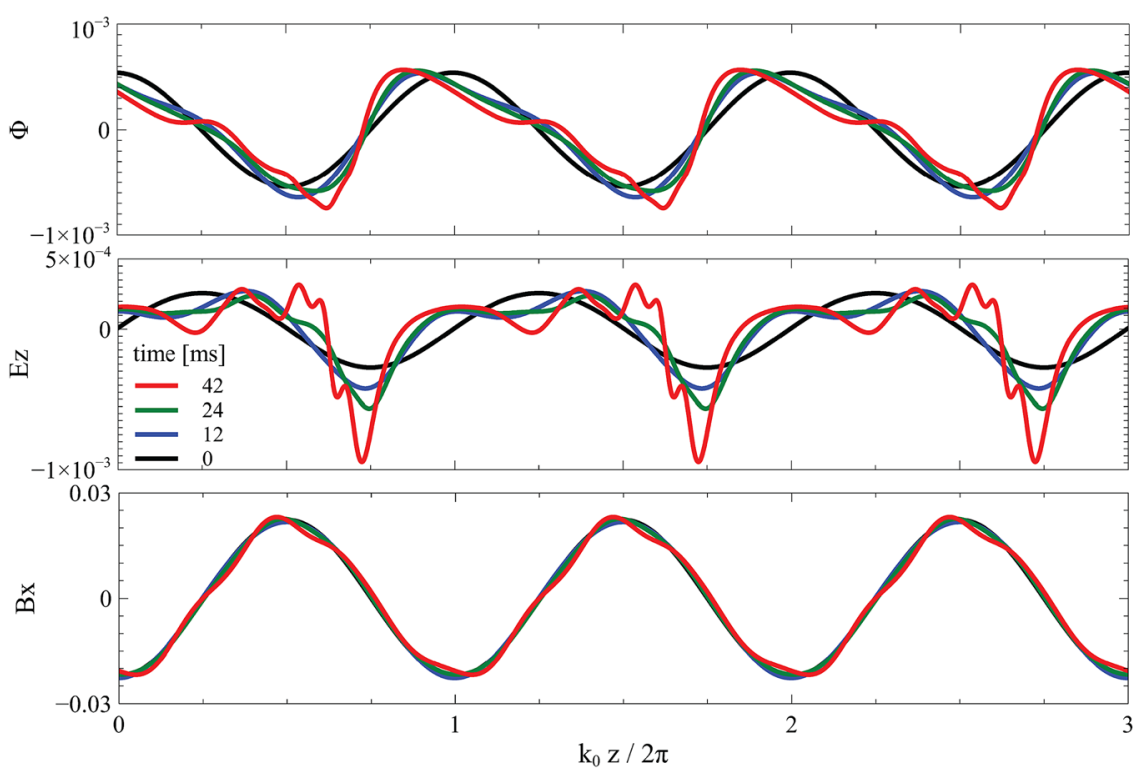

FIG. 5. The evolution of initially monochromatic whistler wave with a realistic amplitude and frequency $f_{0}$ around the first crossover frequency $\sim 0.2 f_{c}$. The simulation results are presented in the whistler wave reference frame (propagating with the whistler wave phase velocity with respect to ions). The electrostatic potential and electrostatic field are normalized to $m c^{2} / e$ and $m c^{2} / e d_{e}$, where $d_{e}=c / 2 \pi f_{p}$ is the electron inertial length. The magnetic field $B_{x}$ is normalized to the background magnetic field $B_{0}$. The electromagnetic field components $E_{x}, E_{y}$ and $B_{y}$ have profiles identical to $B_{x}$ up to a shift in phase.

in phase with the fundamental wave due to the linear dispersion relation at long wavelengths. Similarly, the whistler wave $\left(f_{0}, k_{0}\right)$ produces electron-acoustic waves $\left(\ell f_{0}, \ell k_{0}\right)$ that are in phase until $\left(\ell k_{0}\right)^{-1}$ becomes comparable to the dispersive scale of the electron-acoustic mode that is the Debye length $\lambda_{D}=\left(T_{h} / 4 \pi n_{0} e^{2}\right)^{1 / 2}$. Therefore, the number of harmonics may not exceed $\ell \sim\left(k_{0} \lambda_{D}\right)^{-1} \sim$ $c\left(T_{h} / m\right)^{-1 / 2} \sim 10$, which is consistent with the observed spectrum. Because the steepening of long electron-acoustic waves is known to be described by the Korteweg-de Vries equation [25], we can estimate the steepening time of the whistler electrostatic field as $\tau_{s} \sim 2 A\left(m T_{h}\right)^{1 / 2}\left(e E_{0}\right)^{-1}$, where $E_{0}$ is the initial amplitude of the whistler electrostatic field and $A^{-1}=\left(n_{h} / n_{l}\right)^{1 / 2}\left(3+n_{l} / n_{h}\right)$ [24,25]. Assuming the typical amplitude $E_{0} \sim 10 \mathrm{mV} / \mathrm{m}$, we find $\tau_{s} \sim 50 \mathrm{~ms}$, which is consistent with the simulation results. The slight distortions of the electromagnetic components in the simulations appear because of the high-frequency perturbations of the current densities of the low- and high-energy electrons.

After about $60 \mathrm{~ms}$, the energy leaks out of the steepening region due to the electron-acoustic wave dispersion converting the electric field spikes into oscillations that are inconsistent with observations. The spikes remain persistent in the presence of some dissipation mechanism; e.g., the collisional Burgers dissipation [12] provides persistency of the spikes. In collisionless plasma of the radiation belts, the dissipation is provided not by collisions but by resonant wave-particle interactions, which do not have straightforward inclusion into our hydrodynamic approach. The wave-particle interaction effects were originally included into the hydrodynamic description of ion-acoustic waves [26], and it has been confirmed in simulations of electron-acoustic [24] and Alfvén [27] wave steepening that the collisionless dissipation is capable of providing the persistency of the electric field spikes and magnetic field pulses.

A whistler wave can efficiently exchange energy with electrons with the parallel velocity $v_{\|}$, satisfying the resonance condition, $\omega-k_{\|} v_{\|}=n \omega_{c}$, where $\omega=2 \pi f$, $\omega_{c}=2 \pi f_{c}, \quad k_{\|}=k \cos \theta$ and $n=0, \pm 1, \ldots$ [28]. The steepening of the whistler electrostatic field allows wave energy to cascade to higher frequencies and wave numbers, opening the door for many more resonances: $\omega-k_{\|} v_{\|}=$ $n \omega_{c} / \ell$. This facilitates the efficient energy transfer from the cyclotron resonant electrons, driving the whistler wave, to other electron populations; in particular, to lower energy electrons.

In summary, whistler waves propagating in the twotemperature electron plasma can exhibit the classical steepening producing pronounced spikes in the whistler electrostatic field. The steepening occurs due to the whistler wave coupling to the electron-acoustic mode around the crossover frequency that depends on the background plasma parameters. We have demonstrated the steepening of monochromatic whistler waves with a frequency close to the crossover frequency. In reality, whistler waves are generated in the form of wave packets, and the effect of the coupling will be noticeable for whistler wave packets with non-negligible wave power around the crossover frequency $f$ that satisfies 
$4 \pi n_{l} T_{h} / B_{0}^{2} \sim f / f_{c} \cos \theta-\left(f / f_{c} \cos \theta\right)^{2}$. The steepening explains surprising observations of whistler waves with significant electric field power at harmonics of the whistler wave's fundamental frequency in the Van Allen radiation belts.

The work of I. V., O.A., F. M., and J.W. B. was performed under JHU/APL Contract No. 922613 (RBSP-EFW).

*ivan.vasko@ssl.berkeley.edu

[1] R. M. Thorne et al., Nature (London) 504, 411 (2013).

[2] F. S. Mozer, O. Agapitov, V. Krasnoselskikh, S. Lejosne, G. D. Reeves, and I. Roth, Phys. Rev. Lett. 113, 035001 (2014).

[3] S. P. Gary, O. Chang, and J. Wang, Astrophys. J. 755, 142 (2012).

[4] V. Petrosian and S. Liu, Astrophys. J. 610, 550 (2004).

[5] B. Van Compernolle, J. Bortnik, P. Pribyl, W. Gekelman, M. Nakamoto, X. Tao, and R. M. Thorne, Phys. Rev. Lett. 112, 145006 (2014).

[6] B. Van Compernolle, X. An, J. Bortnik, R. M. Thorne, P. Pribyl, and W. Gekelman, Phys. Rev. Lett. 114, 245002 (2015).

[7] C. F. Kennel and H. E. Petschek, J. Geophys. Res. 71, 1 (1966).

[8] Y. Omura et al., in Dynamics of the Earth's Radiation Belts and Inner Magnetosphere, edited by D. Summers, I. U. Mann, D. N. Baker, and M. Schulz (American Geophysical Union, Washington, D. C., 2013) pp. 243-254.
[9] R. M. Thorne, B. Ni, X. Tao, R. B. Horne, and N.P. Meredith, Nature (London) 467, 943 (2010).

[10] O. Santolík et al., J. Geophys. Res. 108, 1278 (2003).

[11] R. A. Helliwell, Whistlers and Related Ionospheric Phenomena (Stanford University Press, Stanford, 1965).

[12] L. D. Landau and E. M. Lifshitz, Course of Theoretical Physics (Pergamon Press, Oxford, 1959).

[13] R. Z. Sagdeev, Rev. Plasma Phys. 4, 23 (1966).

[14] Yoon, V. S. Pandey, and D.-H. Lee, J. Geophys. Res. 119, 1851 (2014).

[15] C. Cattell et al., Geophys. Res. Lett. 35, L01105 (2008).

[16] P. J. Kellogg, C. A. Cattell, K. Goetz, S. J. Monson, and L. B. Wilson, Geophys. Res. Lett. 37, L20106 (2010).

[17] D. Malaspina et al., J. Geophys. Res., DOI: 10.1002/ 2017JA025005 (2018).

[18] B. U. Ö. Sonnerup and M. Scheible, ISSI Scientific Reports Series 1, 185 (1998).

[19] J. R. Wygant et al., Space Sci. Rev. 179, 183 (2013).

[20] C. A. Kletzing et al., Space Sci. Rev. 179, 127 (2013).

[21] H. O. Funsten et al., Space Sci. Rev. 179, 423 (2013).

[22] K. Watanabe and T. Taniuti, J. Phys. Soc. Jpn. 43, 1819 (1977).

[23] S. P. Gary and R. L. Tokar, Phys. Fluids 28, 2439 (1985).

[24] C. S. Dillard, I. Y. Vasko, F. S. Mozer, O. V. Agapitov, and J. W. Bonnell, Phys. Plasmas 25, 022905 (2018).

[25] R. L. Mace, S. Baboolal, R. Bharuthram, and M. A. Hellberg, J. Plasma Phys. 45, 323 (1991).

[26] E. Ott and R. N. Sudan, Phys. Fluids 12, 2388 (1969).

[27] M. V. Medvedev, P. H. Diamond, V. I. Shevchenko, and V. L. Galinsky, Phys. Rev. Lett. 78, 4934 (1997).

[28] D. Shklyar and H. Matsumoto, Surv. Geophys. 30, 55 (2009). 